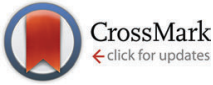

Cite this: Phys. Chem. Chem. Phys., 2016, 18, 26444

Received 1st July 2016,

Accepted 30th August 2016

DOI: $10.1039 / c 6 c p 04607 j$

www.rsc.org/pccp

\section{Metallic nanowires and mesoscopic networks on a free surface of superfluid helium and charge-shuttling across the liquid-gas interface}

\author{
P. Moroshkin, ${ }^{a}$ R. Batulin, ${ }^{\text {ab }}$ P. Leiderer ${ }^{c}$ and K. Kono ${ }^{\text {abd }}$
}

\begin{abstract}
We investigate the motion of electrically charged metallic nano- and microparticles produced by laser ablation in He gas and injected into superfluid helium. In the presence of a vertical static electric field, the particles either perform a repetitive shuttle-like motion transporting the charge across the liquid-gas interface or become trapped under the free surface of liquid He and coalesce into long filaments and complex two-dimensional mesoscopic networks. A classical electrohydrodynamic model is used to describe the motion of charged microparticles in superfluid He. The resulting filaments and networks are analyzed using electron microscopy. It is demonstrated that each filament is in fact composed of a large number of nanowires with a characteristic diameter of order of $10 \mathrm{~nm}$ and extremely large aspect ratios.
\end{abstract}

\section{Introduction}

Laser ablation of metals in various liquids is a well-known method of creating nanoparticles. In many such experiments, it was observed that the produced nanoparticles aggregate into larger structures: nano-necklaces and nano-networks.

Already first experiments ${ }^{1,2}$ on impurity atoms and molecules in superfluid helium have shown that laser ablation of various metals in bulk liquid He leads to formation of filaments with large aspect ratios. Similar filaments and chains of microparticles have been also observed upon injection of solid hydrogen particles into superfluid helium. ${ }^{3-5}$ Those filaments have a typical diameter of some tens or hundreds of microns and could be observed by naked eye. Some filaments possess branches ${ }^{1,6}$ and may form networks or webs with typical dimensions of several millimeters or centimeters. ${ }^{6}$ It was then demonstrated $^{6}$ that the metallic filaments in fact consist of a very large number of twisted and entangled nanowires with typical diameters of 2-20 nm. Their crystalline structure, thermal stability, and electric properties have been investigated in several recent experiments. ${ }^{7-13}$ Similar nanowires have been obtained in experiments $^{14-17}$ with superfluid He micro- and nanodroplets doped with metallic atoms and clusters.

\footnotetext{
${ }^{a}$ RIKEN, Center for Emergent Matter Science, 2-1 Hirosawa, Wako, 351-0198 Saitama, Japan. E-mail: petr.moroshkin@riken.jp; Web: http://www.riken.jp/en/ research/labs/cems/qtm_inf_electron/qtm_condens_phases/

${ }^{b}$ Institute of Physics, Kazan Federal University, Kremlyovskaya st. 18, 420008 Kazan, Russia

${ }^{c}$ Department of Physics, University of Konstanz, Universitätstrasse 10, 78464 Konstanz, Germany

${ }^{d}$ Institute of Physics, National Chiao Tung University, Hsinchu 300, Taiwan
}

Superfluid helium is a peculiar fluid that differs from all other known liquids in many aspects. It has extremely high heat conductivity, low density and surface tension, vanishing viscosity, and supports frictionless motion. Of particular importance is the existence of long-living superfluid vortices with quantized circulation and non-superfluid core of sub-atomic diameter. ${ }^{18}$ Their ability to attract and trap small particles is well known since the pioneering work ${ }^{19}$ on the trapping of free electrons in 1970-ies. It was therefore suggested that in superfluid He both, hydrogen filaments ${ }^{3,5}$ and metallic nanowires ${ }^{6,7}$ are formed due to the aggregation of dopants trapped at the vortex core. Currently, most publications on this quickly growing field of research support that interpretation, ${ }^{10,14-16,20}$ both for bulk superfluid He and for He microdroplets.

The interest in this phenomenon is twofold. First, the nanowires obtained by this novel technique may possess physical properties strikingly different from those obtained by more conventional methods. Second, the structure of the nanowires and their networks may provide new insights into the physics of quantized vortices in infinitely large (bulk) and finite-size (droplet) superfluid systems.

Vortex trapping of individual particles, such as electrons and ions, was extensively studied theoretically. ${ }^{21,22}$ More recently, the diffusion of neutral impurity particles in liquid $\mathrm{He}$ and their coalescence into small clusters has been analyzed. ${ }^{17,20}$ However, a detailed theory of a nanowire or a filament growth on the superfluid He vortex does not yet exist. Especially puzzling is the advanced stage of the filament formation process, when the individual nanowires become entangled and twisted together to form millimeter-sized "ropes" and networks. 
Two observations made already in ref. 6 and reproduced in subsequent studies seem to contradict this model. First, many observed filaments and nanowires have a very rich structure with multiple branches that cannot form on a single vortex. Second, nanowires were formed also in normal fluid $\mathrm{He}$, at a temperature above $2.17 \mathrm{~K}$. However, in that case no larger structures, such as networks and millimeter-long filaments could be observed and the nanowires had a less smooth structure. $^{20}$

In the present work we investigate the formation of nanowires, filaments and networks in a system that represents a two-dimensional trap for impurity particles, as opposed to a one-dimensional trap produced by a quantized vortex. The trapping potential is created by a combination of a vertical static electric field and a horizontal free surface of superfluid He. The trap is loaded by charged metallic micro- and nanoparticles created by laser ablation in He vapor above superfluid He. The resulting mesoscopic structures and their dynamics are observed by collecting the laser light scattered by the injected particles and are subsequently studied by scanning electron microscopy (SEM) at room temperature. In this way we expand the existing studies of metallic nanostructures in superfluid He aiming at a better understanding of the advanced stage of the impurity coalescence process on the mesoscopic scale.

\section{Experimental setup}

The experiments are performed in a helium-bath cryostat with optical access via four side-windows and a window in the bottom. It is cooled to $T=1.4 \mathrm{~K}$ by pumping on the liquid $\mathrm{He}$ in the bath. The sample cell is immersed in the He bath and is filled by condensing He gas from a high pressure gas cylinder. The cell has a cylindrical shape, with a height of $7 \mathrm{~cm}$ and inner diameter of $5.4 \mathrm{~cm}$. A vertical cut through the cell is shown in Fig. 1. Metallic particles are produced by laser ablation of a metal $(\mathrm{Ba}, \mathrm{Cu})$ target with a pulsed, frequency-doubled Nd:YAG laser. The laser produces nanosecond pulses with a repetition rate of 1 to $10 \mathrm{~Hz}$ and a pulse energy of 0.5 to $10 \mathrm{~mJ}$. The laser beam enters the cell via one of the side-windows and hits the vertical target surface. In some experiments, we have also ablated a target, tilted with respect to the vertical axis by approximately 45 degrees, with the working surface tilted

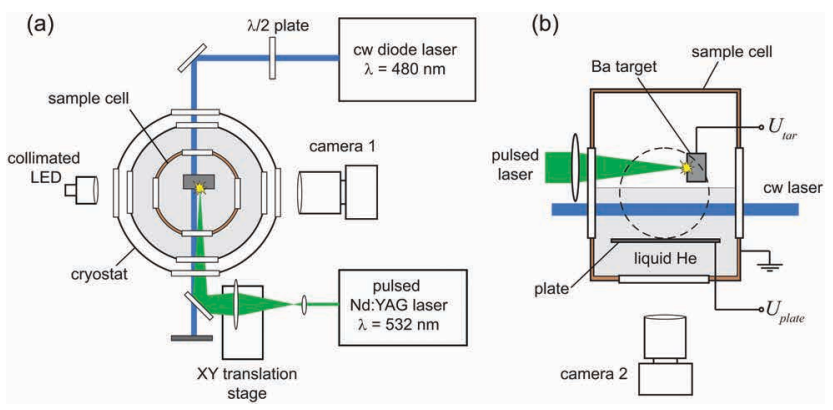

Fig. 1 Experimental setup: (a) top view of the cryostat and optical setup: (b) vertical cross section of the sample cell. towards the liquid. Outside the cryostat, the laser beam is expanded to a diameter of $\approx 3 \mathrm{~cm}$ and then focused by a lens with focal distance of $15 \mathrm{~cm}$, positioned in front of the cryostat window. This lens is mounted on a two-coordinate motorized translation stage that allows motion in the plane orthogonal to the optical axis. During the laser ablation, the lens is moving continuously. The focus of the laser beam is thus shifted along the target surface and every ablation pulse hits a fresh spot. In this way we prevent a hole-drilling effect and obtain a higher stability of the ablation yield.

In the lower part of the cell, $12 \mathrm{~mm}$ below the target, a horizontal plate electrode is mounted. The plate is made either of brass, or of $0.7 \mathrm{~mm}$ thick glass with a transparent conductive coating at the upper surface. The body of the sample cell is electrically grounded. During the experiment, the electric potentials of the target, $U_{\mathrm{tar}}$, and the plate $U_{\text {plate }}$, are set independently.

All cell windows have a clear aperture of $20 \mathrm{~mm}$. The ablation target is positioned in front of the upper part of the window, while the plate can be seen in the lower part. The cell is filled with liquid He up to a certain height, such that the free surface of the liquid is half-way between the target and the plate and can be clearly seen through the side windows. The free surface and the space between the surface and the plate is monitored through a side-window by a digital video camera shown in Fig. 1(a). The camera is oriented either horizontally, or at an angle of $\leq 15^{\circ}$ from below the surface. It is equipped with a macro-lens that has a magnification equal to 1 and a focal distance of $12 \mathrm{~cm}$. The camera is operated at a frame rate of up to $506 \mathrm{fps}$ and allows us to study the fast motion of submillimeter particles and "ropes".

The cell is illuminated either by a white halogen lamp, or by a collimated blue LED. Alternatively, we use the unfocused beam of a cw diode laser with a wavelength $\lambda=480 \mathrm{~nm}$. The latter is aligned orthogonal to the camera and allows us to observe the large-angle light scattering by the particles on a dark background. The direction of the linear polarization of the laser is adjusted by a $\lambda / 2$ plate in order to maximize the light scattering efficiency.

In order to monitor the free surface of liquid He in the cell from below, the transparent bottom electrode is used and the camera is mounted below the cryostat, as shown in Fig. 1(b). The cw laser beam is aligned along the liquid surface. It is expanded up to a diameter of $10 \mathrm{~mm}$ and then focused in the vertical direction by a cylindrical lens to produce a horizontally oriented beam cross-section.

All metallic particles and filaments produced either in bulk superfluid $\mathrm{He}$, or in the gas above it, or at the gas-liquid interface eventually fall on the cell bottom at the end of the experiment, when the liquid He is evaporated. However, during the experiment some filaments may stick to the vertical walls of the cell. Indeed, after the experiment, when opening the cell, we see a lot of deposites on the side wall. The density is particularly high along the horizontal line marking the position of liquid He level during the experiment. The ablation products are collected for the subsequent study on several Si wafers placed inside the cell. The horizontally-oriented wafers are 
placed on the plate electrode, similar to earlier experiments. ${ }^{6}$ The vertically oriented wafers are attached to the side walls of the cell, between the plate and the target. The orientation of filaments found on those substrates allows one to distinguish surface-bound structures from those produced in the bulk.

\section{Results}

First, we have verified that the laser ablation of copper in the sample cell completely filled with superfluid helium leads to the formation of the same kind of nanowires and large networks as reported earlier. ${ }^{6,8}$ In the following we concentrate on the experiments with the ablation target (either $\mathrm{Ba}$ or $\mathrm{Cu}$ ) positioned in He gas above superfluid He.

\section{A. Moving particles}

With $U_{\text {tar }}=+100 \mathrm{~V}$ and $U_{\text {plate }}=-300 \mathrm{~V}$, every ablation laser pulse initiates a shower of positively charged particles moving in superfluid He towards the plate electrode. The real particle size, most likely, is not resolved. The apparent particle diameter varies from 1 pixel $(14 \mu \mathrm{m})$ to 10 pixel. Note that the depth of focus of the lens is smaller than the illuminated area. Therefore, not all observed particles produce sharp images.

Most particles $(\approx 85 \%)$ move with constant velocity in the range of $0.5-10 \mathrm{~cm} \mathrm{~s}^{-1}$. The velocities are determined by comparing the positions of the same particle in successive frames in the video using the program Diatrack. A histogram showing the velocity distribution of down-going particles at $U_{\text {tar }}=+100 \mathrm{~V}, U_{\text {plate }}=-300 \mathrm{~V}$ is shown in Fig. 2(a).

Some particles also move upwards. Down-going and up-going particles have been observed under both polarities of the applied voltage. The velocity distribution of up-going particles for
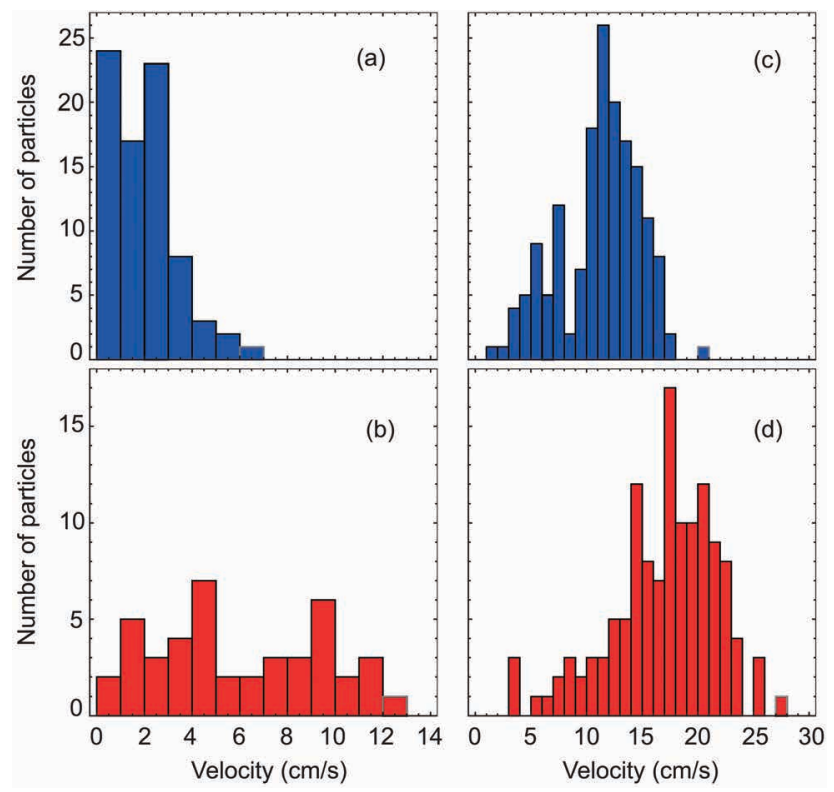

Fig. 2 Velocity distributions of Ba particles moving downwards (a), (c) and upwards (b), (d) in a static electric field. (a), (b) $U_{\text {tar }}=+100 \mathrm{~V}, U_{\text {plate }}=-300 \mathrm{~V}$; (c), (d) $U_{\text {tar }}=-200 \mathrm{~V}, U_{\text {plate }}=+600 \mathrm{~V}$.
$U_{\text {tar }}=+100 \mathrm{~V}, U_{\text {plate }}=-300 \mathrm{~V}$ is plotted in Fig. 2(b). At this relatively low electric field strength their number is much smaller than the number of down-going particles.

In a stronger electric field, the distribution of the particle velocities becomes broader and its maximum shifts towards larger velocities. This effect is illustrated by the velocity distributions at $U_{\mathrm{tar}}=-200 \mathrm{~V}, U_{\text {plate }}=+600 \mathrm{~V}$, shown in Fig. 2(c) and (d). Under these conditions, the number of up-going particles is almost the same as the number of down-going particles. Interestingly, the velocity distribution of particles moving upwards is peaked at a larger velocity than that of the particles moving down under the same conditions, at either polarity of the applied voltage.

We notice that many of those particles perform a repetitive shuttle-like motion between the free liquid He surface and the electrode. The particle touches the free surface and moves down at some constant speed, $v_{\text {down }}$. After touching the bottom electrode, the particle returns to the surface also at a constant, but somewhat different speed, $v_{\text {up }}$, and the whole cycle repeats many times. The effect is much more pronounced with the positive potential of the plate electrode and negative potential of the target than at the opposite polarity. Typically, for each particle $v_{\text {up }}>v_{\text {down }}$ and both velocities are reproduced in the subsequent cycles. The motion of one such shuttle-particle at $U_{\text {tar }}=-200 \mathrm{~V}, U_{\text {plate }}=+600 \mathrm{~V}$ is illustrated in Fig. 4 that shows the vertical coordinate of the particle as a function of time. $Z=0$ corresponds to the free surface of the liquid. The bottom electrode is $9 \mathrm{~mm}$ below. Only the upper part of the trajectory $(Z>-3 \mathrm{~mm}$ ) was illuminated by the blue laser and captured by the camera. Nevertheless, linear extrapolations of $Z(t)$ nicely reproduce the whole trajectory. For this particle, $v_{\text {up }}=19 \pm 2 \mathrm{~cm} \mathrm{~s}^{-1}$, $v_{\text {down }}=16 \pm 1 \mathrm{~cm} \mathrm{~s}^{-1}$, and one full cycle takes $105 \pm 5 \mathrm{~ms}$.

In Fig. 3 we show the histograms of the appearance time, $T_{0}$ of the up-going and down-going particles with respect to the ablation laser pulse. Here, $T_{0}$ corresponds to the beginning of the particle trajectory within the field of view of the camera.

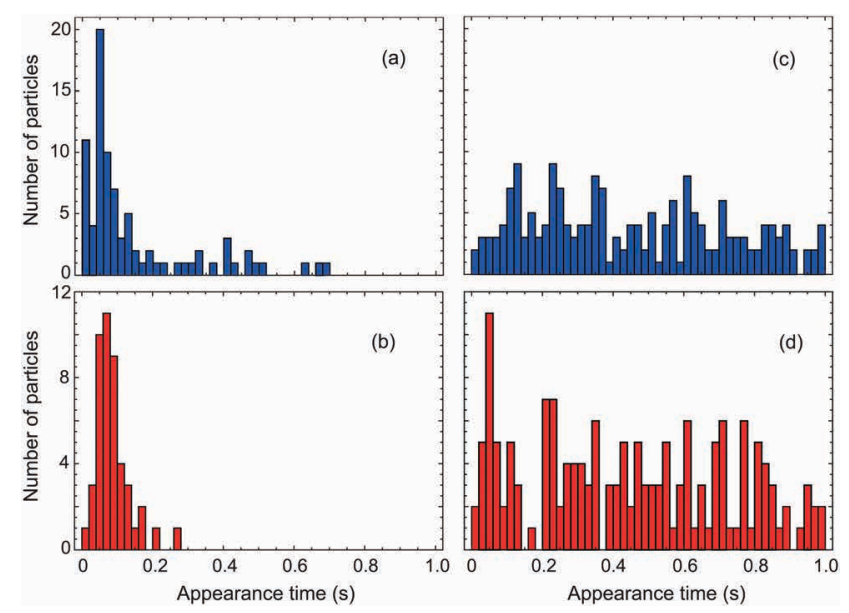

Fig. 3 Distributions of the appearance times of Ba particles moving downwards (a), (c) and upwards (b), (d) in a static electric field. (a), (b) $U_{\text {tar }}=$ $+100 \mathrm{~V}, U_{\text {plate }}=-300 \mathrm{~V}$; (c), (d) $U_{\text {tar }}=-200 \mathrm{~V}, U_{\text {plate }}=+600 \mathrm{~V}$. The ablation laser pulse is fired at $t=0$. 


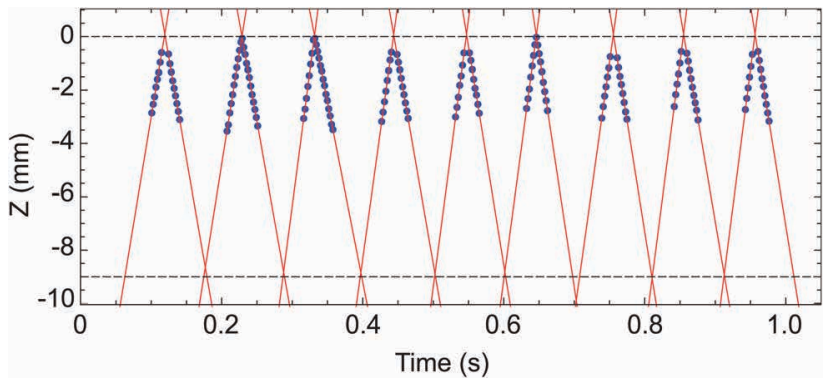

Fig. 4 Vertical displacement of a single particle performing a shuttle-like motion between the free surface of superfluid $\mathrm{He}$ and the bottom electrode. Free surface is at $Z=0$, plate electrode at $Z=-9 \mathrm{~mm}, U_{\mathrm{tar}}=$ $-200 \mathrm{~V}, U_{\text {plate }}=+600 \mathrm{~V}, T=1.4 \mathrm{~K}$. Every data point corresponds to a frame of a video recording, solid lines - linear fits to the data.

For the particles performing the shuttle-like motion, every passage through the field of view is counted as a new trajectory.

At $U_{\text {tar }}>0, U_{\text {plate }}<0$, most particles appear only once, or perform only one cycle between the two successive laser pulses. They appear mostly within $50 \mathrm{~ms}$ after the ablation laser pulse. As one can see in Fig. 3(a) and (b), the motion of the particles from below and from above starts approximately at the same time. Some number of particles stays at the bottom before the ablation pulse and are detached from the electrode by the laser pulse. After arriving at the free surface, some of them return back and are observed for the second time. These returning particles produce a second, smaller peak in Fig. 3(a) at $T_{0} \approx 0.4 \mathrm{~s}$.

The mechanism detaching the particles from the bottom electrode remains unclear. Most likely, the particles are shaken off by a sound wave or a mechanical shock generated by the laser pulse hitting the target above the liquid. The detachment can be also induced by the turbulence created by a shower of positive ions moving down after the ablation laser pulse. Atomic ions: $\mathrm{He}^{+}, \mathrm{Ba}^{+}, \mathrm{Cu}^{+}$possess much larger mobilities than the larger particles observed here. They are expected to reach the bottom electrode within several milliseconds after the ablation pulse. A similar detachment effect is observed under the stronger electric field of the same polarity. In that case, the particles move faster in both directions and the delay between their appearance time and the laser pulse becomes shorter.

At $U_{\text {tar }}<0, U_{\text {plate }}>0$, the particle motion continues during the whole $1 \mathrm{~s}$ time interval between the successive laser pulses. At a large electric field strength, most particles move at a speed of $10-20 \mathrm{~cm} \mathrm{~s}^{-1}$ and typically make several cycles within this time interval. Their appearance times therefore are distributed more uniformly from 0 till $1 \mathrm{~s}$, as shown in Fig. 3(c) and (d). After switching the ablation laser off, the motion continues for another $\approx 3$ seconds.

In addition to the point-like particles, we observe elongated objects with apparent aspect ratios between 1 and 50. As discussed above, the real transversal (shorter) diameter of these particles may not be resolved by the camera. Only the length of the particle can be determined since it is certainly not diffraction-limited. We also make a correction for the apparent elongation of the particles due to the distance traveled by the particle within a single exposure time $(2 \mathrm{~ms})$. The resulting particle lengths are in the range of 40 to $200 \mu \mathrm{m}$. The elongated particles move between the bottom electrode and the free surface of superfluid He in a manner similar to that of point-like particles, described above.

The observations reported above imply that the particles carrying electric charge are trapped under the free surface of liquid helium by a combined action of the electric force that pushes them upwards and the surface tension that prevents them from leaving the liquid. The particle touching the surface is bombarded by the ions of the opposite polarity produced in a large amount by the laser ablation above the liquid. The charge of the particle thus is neutralized and is replaced by the charge of the opposite sign. The particle then starts moving down, towards the plate electrode. Upon reaching the plate, the particle transfers its charge to it, acquires some charge of the opposite sign and is driven back to the surface. In the process, the particle grows larger due to the attachment of charged particles arriving from above.

\section{B. Filaments and networks}

With a positive potential applied to the target and negative potential at the plate, impurity particles of a new type can be observed in the sample cell. There are filaments with diffraction-limited width and a length of order of a millimeter, or even longer. The first filaments appear after some 10-20 minutes of laser ablation. They are aligned either vertically, along the electric field lines, or horizontally, along the free surface of the liquid.
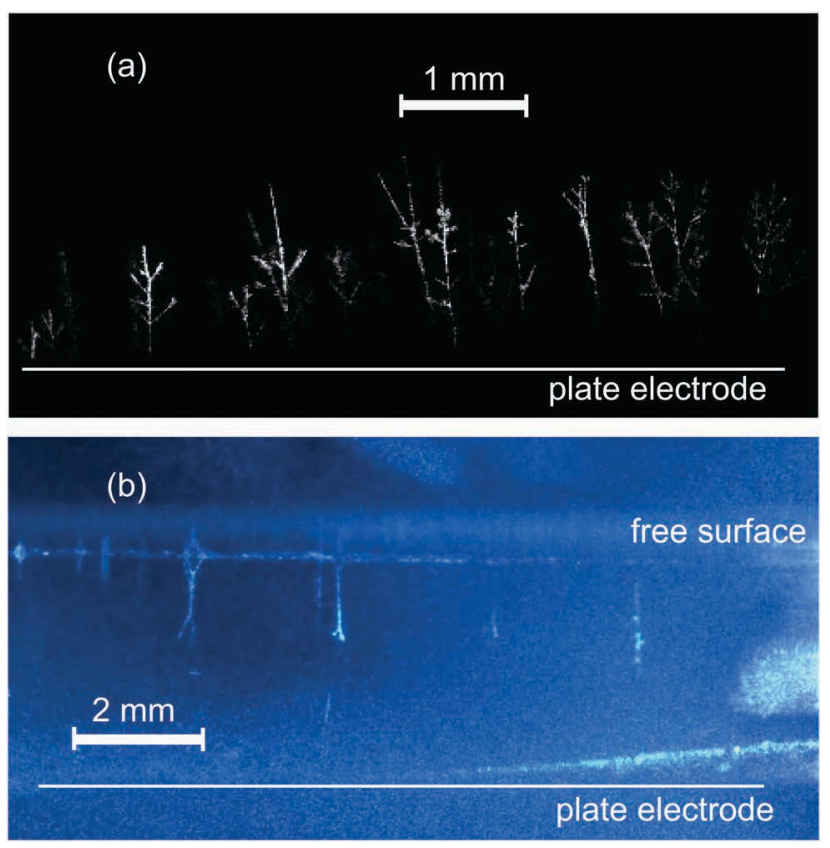

Fig. 5 Images of vertically oriented $\mathrm{Ba}$ filaments in superfluid $\mathrm{He}$. (a) filaments pinned at the bottom electrode; (b) filaments attached to the free surface of liquid He. $U_{\text {tar }}=+300 \mathrm{~V}, U_{\text {plate }}=0, T=1.5 \mathrm{~K}$. Pictures are taken via a side window. Illumination by a cw blue laser. The photo in (b) is made by a color camera different from the camera described in Section II. 
First, we discuss the vertically aligned filaments. Fig. 5 shows some typical images. Some of the filaments have their lower ends attached to the bottom electrode and stretch upwards, as shown in Fig. 5(a). Other filaments shown in Fig. 5(b) have the upper ends attached at the free surface of the liquid and the lower ends hanging in the liquid. The filaments bound to the liquid surface can freely move in the horizontal direction without changing their vertical orientation. They are electrically charged and occasionally leave the surface and move down. Upon touching the bottom electrode by their lower end, they either stick to it, or change the direction of their motion and return to the surface. The frequency of these events depends on the strength of the electric field and the repetition rate of the ablation laser pulses. Under some conditions, we observe periodic oscillations of one or several vertically aligned filaments between the electrode and the free surface of liquid He.

Many of the vertical filaments have side branches (Fig. 5(a)). As the ablation is continued, the main filament and its branches grow longer and the number of branches increases. Eventually, the structure evolves into a "tree" with numerous primary, secondary etc. branches. The "root" of this "tree" is usually pinned at the bottom electrode, its trunk is oriented vertically, and the upper part is reaching the free surface of the liquid.

Besides the vertically oriented filaments and "trees", we also observe filaments floating on the free surface of liquid He and oriented horizontally, parallel to the surface. In fact, their number is much larger than that of the vertically oriented structures. Occasionally, some of those filaments leave the surface, change the orientation to vertical and move towards the electrode. We also observed the inverse process: a vertical filament coming from the bottom towards the surface, upon reaching the surface may also change the orientation and stretch along the surface. If the ablation continues for a long time, the density of surface-bound filaments becomes very high. They have many branches and form complicated networks and tree-like dendritic structures.

Fig. 6 shows the image of one particularly large network or a "tree" floating at the liquid He surface and photographed from below, via the bottom window. This network was created by approximately 1 hour of laser ablation at a repetition rate of $2 \mathrm{~Hz}$ at $U_{\text {tar }}=+100 \mathrm{~V}, U_{\text {plate }}=0$. One end of this network is permanently attached to the bottom electrode and the whole network is electrically charged. The neighboring branches repel each other and therefore all branches are pointing in different directions. The whole network is practically stationary.

A more complicated behavior is observed when the network is floating at the surface without a permanent electric contact with any electrode. In this case, the flow of positive charge carriers generated by the laser ablation above the liquid and absorbed by the network leads to the gradual neutralization of the negative charge already present at the network. The network starts to shrink: the branches lose their straight linear shape and orthogonality. Parts of the network approach closer together and tend to align parallel to each other. Separate filaments and smaller networks also approach the main network and stick

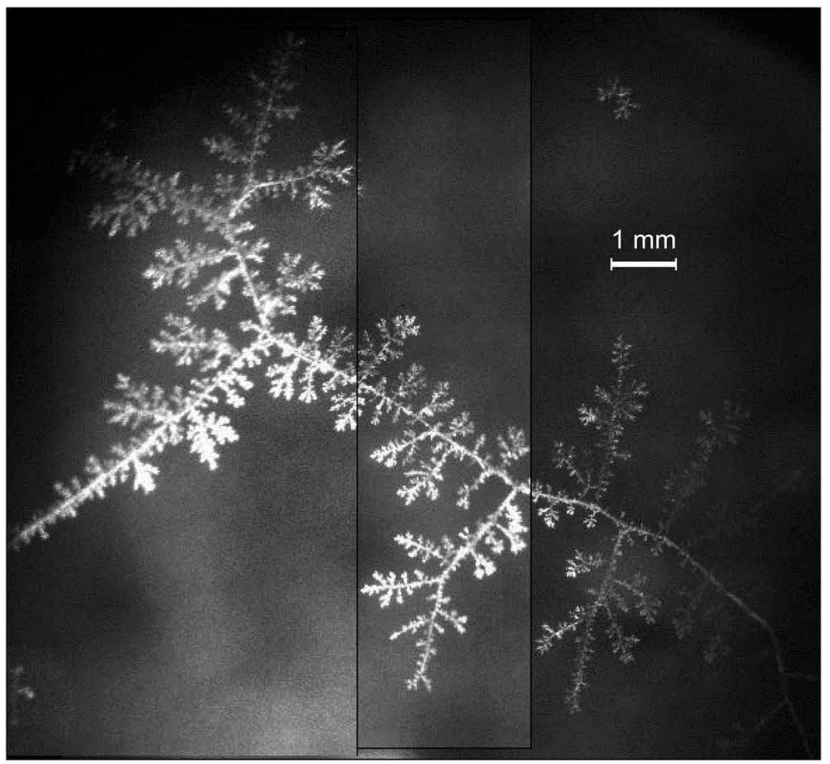

Fig. 6 Image of $\mathrm{Ba}$ network at the free surface of superfluid $\mathrm{He}, U_{\mathrm{tar}}=$ $+100 \mathrm{~V}, U_{\text {plate }}=0, T=1.4 \mathrm{~K}$. Illumination by a cw blue laser, picture is taken via the bottom window of the cryostat and the sample cell.

to it, thus forming an even larger structure. At some point, one end of the network starts to bend down, sinks to the bottom and touches the plate electrode. The network then suddenly becomes charged, all the branches straighten up and the network spreads out. At the same moment, it loses the contact with the bottom electrode and the whole cycle repeats again. The network in the process grows larger by attaching more filaments and subnetworks. This type of dynamics is illustrated in Fig. 7 by the frames from a video recording of one such network that has touched and separated from the bottom electrode at $t=0$. Fig. 7(a) shows the network in the most stretched state, at $t=0.7 \mathrm{~s}$.
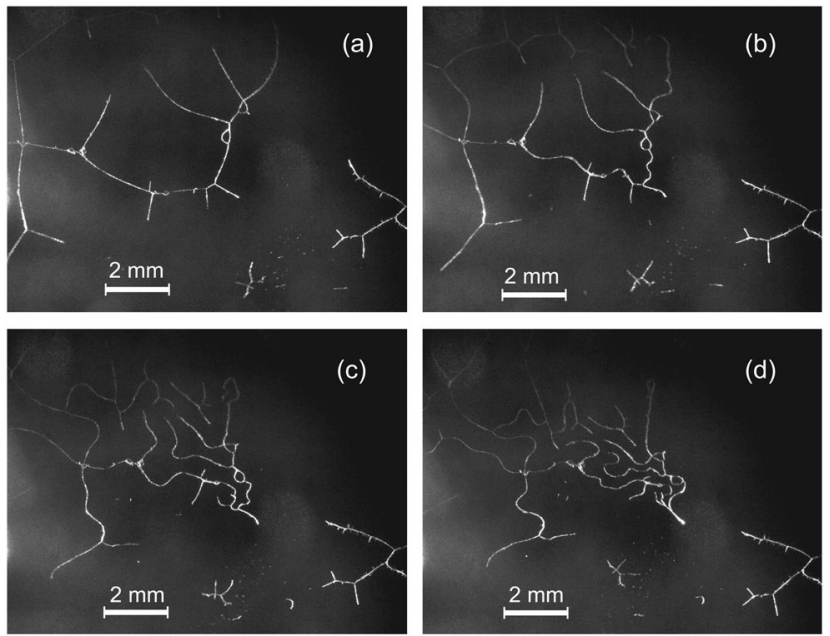

Fig. 7 Series of images illustrating the motion of the same Ba network at the free surface of superfluid $\mathrm{He}, U_{\text {tar }}=+100 \mathrm{~V}, U_{\text {plate }}=0, T=1.4 \mathrm{~K}$. Illumination by a cw blue laser, pictures are taken via the bottom window. (a) Stretched network $0.7 \mathrm{~s}$ after touching the bottom electrode; (b), (c), (d) shrinking network at $t=1.3,1.8$, and $2.3 \mathrm{~s}$, respectively. 
Fig. 7(b), (c), and (d) show the same network in the process of shrinking at $t=1.3,1.8$, and $2.3 \mathrm{~s}$, respectively. The whole cycle in this case has taken $2.8 \mathrm{~s}$.

\section{Laser-induced motion}

Small metallic particles and filaments trapped at the free surface of liquid $\mathrm{He}$ and illuminated by intense $\mathrm{cw}$ blue laser light start to move and accelerate in the direction of the laser beam propagation.

A similar effect could be produced by a cw illumination of the vertical filaments and "trees" pinned at the bottom electrode. The cw laser beam induces rotation of a "tree" around the vertical axis. A long vertically oriented filament bends and tilts in the direction of the laser beam propagation.

We attribute these observations to the effect of the counterflow of superfluid and normal fluid components of superfluid He. The counterflow is induced at the surface of the metallic particle or filament that is heated by the intense laser beam. There is a temperature gradient that leads to the flow of superfluid component towards the warmer spot and the flow of normal component in the opposite direction. The superfluid component is converted into the normal-fluid component at the heated surface. The superfluid component carries the momentum density $P_{\text {sup }}=n_{\text {sup }} \rho_{\mathrm{He}} v_{\text {sup }}$ and the normal-fluid component has the momentum density $P_{\text {norm }}=-n_{\text {norm }} \rho_{\mathrm{He}} v_{\text {norm }}$. Here, $n_{\text {sup }}$ and $n_{\text {norm }}$ are the superfluid fraction and the normal-fluid fraction, respectively, $n_{\text {norm }}+n_{\text {sup }}=1$. $v_{\text {sup }}$ and $v_{\text {norm }}$ are the velocities of the two components and $\rho_{\mathrm{He}}$ is the density of liquid He. The positive direction is along the propagation of the laser beam. Since the total density of the liquid is not changing due to the counterflow, one can write: $n_{\text {sup }} v_{\text {sup }}=$ $n_{\text {norm }} v_{\text {norm}}$. Upon the contact with the particle surface, the momentum density of the liquid is changed by

$$
\Delta P=P_{\text {norm }}-P_{\text {sup }}=-2 \rho_{\mathrm{He}} n_{\text {sup }} v_{\text {sup }}
$$

The momentum of the opposite sign is transferred to the particle that leads to the motion of the particle in the direction of the laser beam propagation. The particle is thus moving towards its own shadow. This observation implies that the laser heating creates a sufficient gradient of temperature within the metallic particle. A homogeneously heated particle would generate a spherically symmetric counterflow with zero total momentum of each component.

\section{Microscopy study}

In liquid $\mathrm{He}$, both $\mathrm{Cu}$ and $\mathrm{Ba}$ particles behave very similar and produce the same kind of filaments and networks. When exposed to the atmosphere, Ba networks quickly oxidize. Under the microscope, they look as strings of oxide micro-particles of irregular shapes. We therefore concentrate in this section on the $\mathrm{Cu}$ networks that are sufficiently robust.

Typical electron microscope (SEM) images of the $\mathrm{Cu}$ filaments collected at the surface of superfluid He are shown in Fig. 8 and 9. In the upper row of Fig. 8 we present two low magnification images, $120 \times 90 \mu \mathrm{m}$ showing several very long filaments. The second row of images in the same figure shows
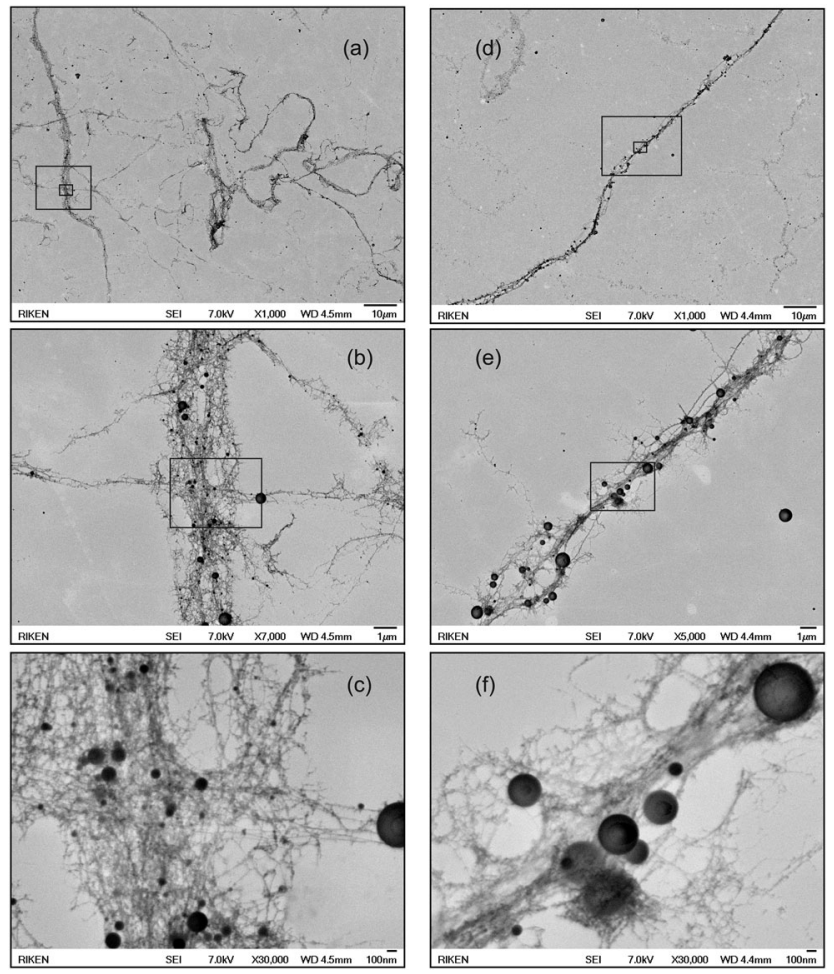

Fig. 8 Electron microscope (SEM) images of Cu filaments collected at the surface of superfluid He. (a) Low magnification image $120 \times 90 \mu \mathrm{m}$; (b) zoom into the left side of (a), $17 \times 13 \mu \mathrm{m}$; (c) zoom into the central part of (b), $4 \times 3 \mu \mathrm{m}$; (d) low magnification image $120 \times 90 \mu \mathrm{m}$; (e) zoom into the central part of (d), $24 \times 18 \mu \mathrm{m}$; (f) zoom into the central part of (e), $4 \times 3 \mu \mathrm{m}$. Black rectangles mark the zoom area.

the results of a zoom into two of those filaments. Finally, the two pictures of the third row show the high magnification zoom into the same filaments. In these pictures one can clearly see that the filaments are composed of a large number of entangled nanowires. The typical width of a single nanowire is of order of $10 \mathrm{~nm}$ and is most likely limited by the resolution of the microscope. Note also a number of spherical nanoparticles. Most of them are attached to the filaments in random places, although there are also free nanoparticles. The size distribution of those spherical nanoparticles is shown in Fig. 10. It is peaked at $60-80 \mathrm{~nm}$ and has a rather long tail at large particle diameters.

Fig. 9 shows high-magnification images taken in different parts of several filaments, where the density of nanowires is not very high. This allows us to see the connections between individual nanowires and between the nanowires and nanoparticles. Note a large number of T-shaped junctions, or forks that certainly could not be created by a crossing of two nanowires randomly falling on the substrate.

\section{Theoretical model}

In this section, the motion of small metallic particles in liquid He is analyzed within a framework of classical electrostatics and hydrodynamics. 

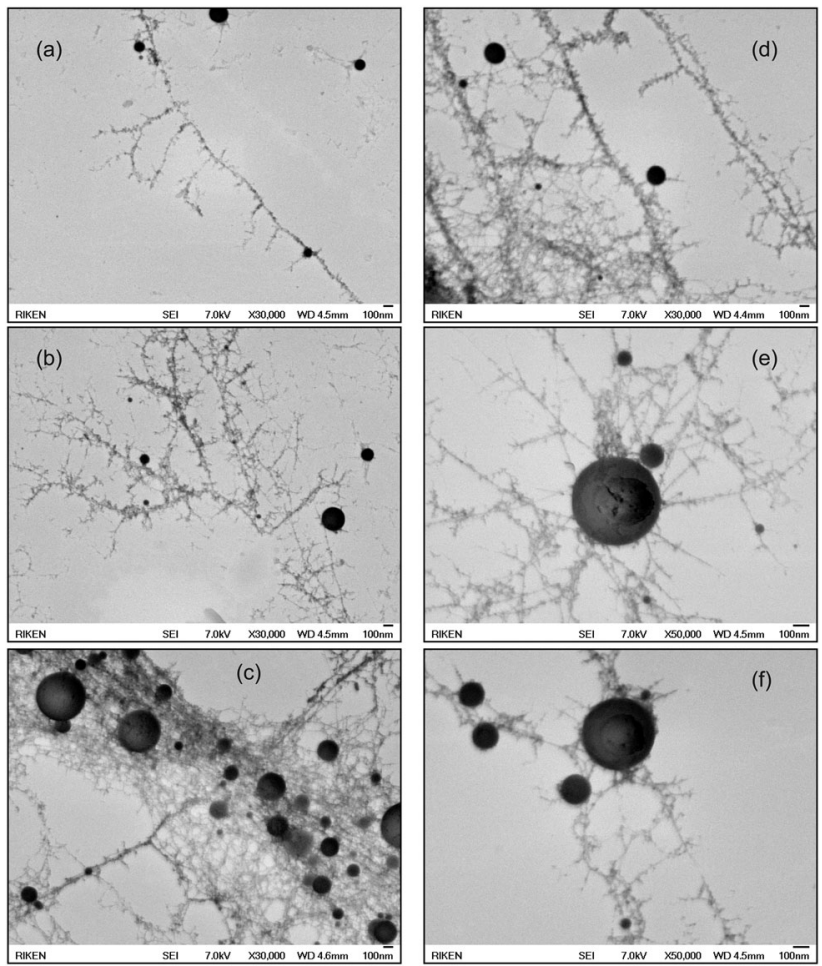

Fig. 9 High magnification electron microscope (SEM) images of $\mathrm{Cu}$ filaments with a large number of branches and T-junctions. (a)-(d) Image size $3 \times 4 \mu \mathrm{m}$; (e) and (f) image size $2.4 \times 1.8 \mu \mathrm{m}$.

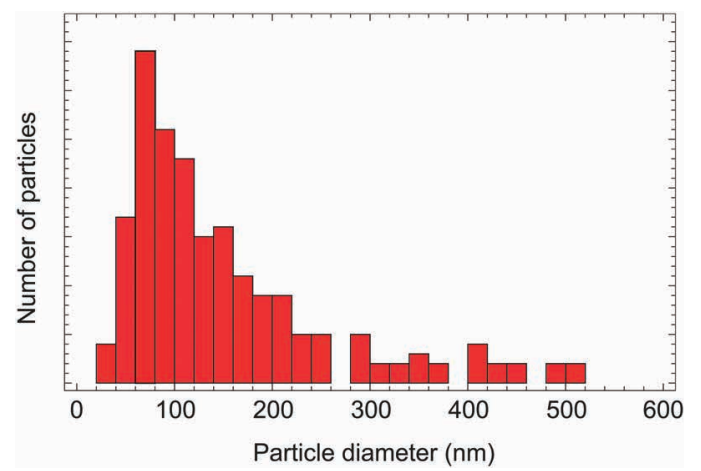

Fig. 10 Nanoparticle size distribution. Histogram of diameters of $\approx 200$ spherical nanoparticles found on the high-magnification SEM images.

\section{A. Shuttle-like particle motion}

Periodic motion of a charged conductive micro-particle or a droplet between two electrodes in a dielectric liquid has been recently reported. ${ }^{23-27}$ The phenomenon is referred to as a contact charge electrophoresis $^{27,28}$ or a charge-shuttling ${ }^{23}$ and was analyzed theoretically in ref. 27 and 28. More work was done on charge transport by larger, sub-millimeter and millimetersized particles of spherical and cylindrical shapes. ${ }^{29-36}$ Some experiments of this kind utilized cryogenic superfluid helium as a dielectric liquid. ${ }^{34,35,37,38}$ In ref. 37 , the motion of spherical particles 10 to 100 micron in diameter was investigated. No visual observation of moving particles was reported. Instead, the authors observed a periodic sequence of pulses of the electric current carried by a single oscillating particle. The authors of ref. 34 and 35 studied the motion of millimeter-sized spherical and cylindrical particles and their influence on the electric breakdown in liquid helium. The motion of charged micronsized dust particles in liquid helium and their recharging in a contact with a positively charged electrode has been reported in ref. 38 .

Two distinct types of particles are present in our experimental system: spherical nanoparticles and nanowire segments of various lengths. We analyze their motion in the following two subsections, mostly using the model developed in ref. 29.

\section{B. Spherical particles}

During the contact between the metal particle and a solid electrode, the particle is charged and acquires the same electric potential as that of the electrode. The charge can be computed by using the method of image charges. ${ }^{39}$ For a spherical particle of radius $R$ one finds:

$$
Q_{\mathrm{s}}=\frac{2 \pi^{3} \varepsilon \varepsilon_{0}}{3} R^{2} E
$$

Here, $\varepsilon$ is the relative permittivity of the liquid and $E$ is the strength of the electric field that is assumed to be uniform far away from the particle. In the experiments, the electric contact between the particle and the electrode is usually established at some small finite distance, via the electric breakdown in the dielectric. The latter is induced by the enhanced electric field in the narrow gap between the plate and the approaching particle.

If the charge is known, one can calculate the electrostatic force pushing the particle away from the electrode. For the particle moving upwards, this force must exceed the gravity minus the buoyancy force. One thus obtains the expression for the threshold or "lifting" field strength $E_{\mathrm{d}}$ :

$$
E_{\mathrm{d}}=\sqrt{\frac{2 g\left(\rho_{\mathrm{Ba}}-\rho_{\mathrm{He}}\right)}{\pi^{2} \varepsilon_{0} \varepsilon} R}
$$

Here, $\rho_{\mathrm{Ba}}=3.51 \mathrm{~g} \mathrm{~cm}^{-3}$ and $\rho_{\mathrm{He}}=0.145 \mathrm{~g} \mathrm{~cm}^{-3}$ are the densities of solid $\mathrm{Ba}$ and liquid $\mathrm{He}$, respectively, and $g$ is the acceleration due to the gravity. Calculations show that particles with a size $R \leq 14 \mu \mathrm{m}$ can be lifted by an electric field of $1 \mathrm{kV} \mathrm{cm}^{-1}$, the strongest field applied in our experiments. At the smallest applied electric field strength, $E=400 \mathrm{~V} \mathrm{~cm}^{-1}$, the largest lifted particle has a radius of $2.2 \mu$. Spherical particles of relevant sizes were indeed found in the SEM study, as reported in Section III D.

In the liquid, the motion of a small particle is strongly damped due to the viscous drag force, $F_{\text {drag }}$.

$$
F_{\text {drag }}=C_{\mathrm{d}} \frac{\pi \rho_{\mathrm{He}}}{2} R^{2} v^{2}
$$

Here, $C_{\mathrm{d}}$ is the drag coefficient that depends on the Reynolds number.

$$
\operatorname{Re}=\frac{2 R \rho_{\mathrm{He}} v}{\eta}
$$

where $\eta$ is the dynamic viscosity of the liquid and $v$ is the particle velocity. For superfluid $\mathrm{He}$, the viscosity is due the 
normal fluid component: $\eta(T)=\eta_{\text {norm }}(T) \times n_{\text {norm }}(T)$. Here, $\eta_{\text {norm }}(T)$ is the viscosity of the normal component and $n_{\text {norm }}(T)$ is the normal-fluid fraction. Both, $\eta_{\text {norm }}(T)$ and $n_{\text {norm }}(T)$ were taken from ref. 40 . For the temperature of $1.4 \mathrm{~K}$, at which most our experiments have been carried out, the viscosity is $\eta=1.1 \times 10^{-7} \mathrm{~Pa}$ s. This value is used in all our calculations discussed below.

At $\operatorname{Re} \lesssim 1, C_{\mathrm{d}}=24 / \mathrm{Re}$ and eqn (4) reduces to the Stokes formula:

$$
F_{\text {drag }}=F_{\mathrm{s}}=6 \pi \eta R v
$$

However, at the experimentally observed velocity of $20 \mathrm{~cm} \mathrm{~s}^{-1}$, the condition $\operatorname{Re} \leq 1$ is not fulfilled for any particle with a radius larger than $2 \mathrm{~nm}$. For $\mathrm{Re}>1$, no analytical relationship exists between the drag coefficient and Re. We therefore use the experimental dependence of $C_{\mathrm{d}}(\mathrm{Re})$ that is shown in ref. 41.

Equating the total force acting on the particle to zero, one obtains the velocity in the Stokes regime:

$$
v_{\mathrm{s}}=\frac{\pi^{2} \varepsilon \varepsilon_{0}}{9 \eta} R E^{2} \pm \frac{2 g\left(\rho_{\mathrm{Ba}}-\rho_{\mathrm{He}}\right)}{9 \eta} R^{2}
$$

Here, the minus sign corresponds to the particle moving upwards and the plus sign corresponds to the particle moving down. For large values of the Reynolds number, the equation for the particle velocity is solved numerically.

We assume that the charge acquired by the particles at the bottom electrode is well described by eqn (2). The experimental observation that the particles moving down have smaller velocities than those moving upwards implies that the charge acquired by the particle at the free surface of liquid $\mathrm{He}$ is smaller than this value. The experimental velocities of the particles moving upwards (see Fig. 2) thus can be compared with those computed using the model outlined above. The calculated electric charge normalized to the value of the elementary charge $e$ is plotted in Fig. 11(a) as a function of the particle radius for several typical values of the electric field strength. The corresponding velocities of up-going particles are plotted in Fig. 11(b).

The calculated velocities lie in the range of $0-10 \mathrm{~cm} \mathrm{~s}^{-1}$ that should be compared with the experimentally observed velocities, $v=1-25 \mathrm{~cm} \mathrm{~s}^{-1}$. For the particles with $R \leq 100 \mathrm{~nm}$, eqn (2) predicts a charge significantly smaller than the elementary charge. Such small particles therefore cannot be charged and should be excluded from the analysis. The minimum velocity of the up-going particles is thus $v_{\text {min }} \approx 1.5 \mathrm{~cm} \mathrm{~s}^{-1}$ at $E=400 \mathrm{~V} \mathrm{~cm}^{-1}$ and $v_{\text {min }} \approx 5 \mathrm{~cm} \mathrm{~s}^{-1}$ at $E=1 \mathrm{kV} \mathrm{cm}^{-1}$.

Under the conditions of our experiments, the calculated particle velocity can not exceed $10 \mathrm{~cm} \mathrm{~s}^{-1}$, for any particle size. This limiting value of the velocity is more than twice lower than the maximum particle speed obtained in the experiment.

\section{Nanowires}

The charge acquired by a cylinder of the length $L$ and radius $r$, oriented orthogonally to the electrode surface ${ }^{39}$ is

$$
Q_{\mathrm{c}}=\frac{\pi \varepsilon \varepsilon_{0}}{\ln (2 L / r)-1} L^{2} E
$$

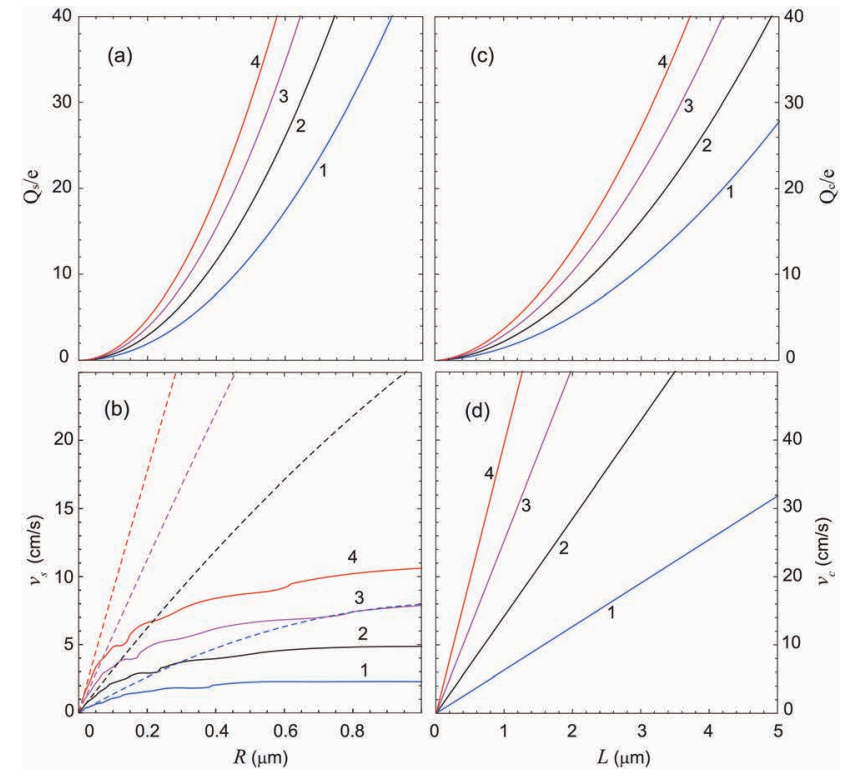

Fig. 11 (a) and (b) Calculated electric charge (a) and velocity (b) of a spherical nanoparticle as a function of the particle radius $R$. (c) and (d) Calculated charge (c) and velocity (d) of a vertically oriented nanowire, as a function of the nanowire length, $r=5 \mathrm{~nm}$. In (b) dashed lines correspond to Stokes limit (low Reynolds number), solid lines - experimental dependence $C_{\mathrm{d}}(\mathrm{Re}){ }^{41}$ In all four plots curve 1 corresponds to $E=400 \mathrm{~V} \mathrm{~cm}^{-1}$, curve 2: $E=600 \mathrm{~V} \mathrm{~cm}^{-1}$, curve $3: E=800 \mathrm{~V} \mathrm{~cm}^{-1}$, curve $4: E=1 \mathrm{kV} \mathrm{cm}^{-1}$. $T=1.4 \mathrm{~K}$, particle and nanowire moving upwards.

Here, the wire is modeled as a half of an ellipsoid, standing upright on the electrode plate. The numerical calculations ${ }^{31,42}$ have shown that the charge acquired by a cylindrical wire with hemispherical or straight cut ends is larger than predicted by eqn (8) by $\approx 50 \%$. Following ref. 29 we use eqn (8) in our calculations.

The strength of the lifting field, $E_{\mathrm{d}}$ is obtained by equating the electric force acting on the wire with the sum of the gravity and buoyancy forces:

$$
E_{\mathrm{d}}=\sqrt{\frac{g\left(\rho_{\mathrm{Ba}}-\rho_{\mathrm{He}}\right) r^{2}}{\varepsilon_{0} \varepsilon L}} \cdot \sqrt{\ln \frac{2 L}{r}-1}
$$

The condition of a laminar flow for a long cylinder is the same as for a spherical particle:

$$
\mathrm{Re}=\frac{2 r \rho_{\mathrm{He}} v}{\eta} \lesssim 1
$$

The data on the dependence of the drag coefficient on the Reynolds number $C_{\mathrm{d}}(\mathrm{Re})$ for wires are much more scarce than for spheres. However, the typical thickness of the nanowires observed in our experiments is $2 r \approx 10 \mathrm{~nm}$, that is much smaller than the typical size of spherical particles. The observed velocities of 1-25 $\mathrm{cm} \mathrm{s}^{-1}$ thus correspond to the Reynolds number in the range of $\mathrm{Re}=0.1-3.0$. We therefore assume that the deviations from the Stokes regime are not too large for the whole range of observed velocities. In this regime, the drag 
force acting on the wire is: ${ }^{29}$

$$
F_{\text {drag }}=F_{\mathrm{c}}=\frac{2 \pi \eta L v}{\ln (L / 2 r)+0.193}
$$

By equating the sum of all forces to zero, we find the expression for the steady velocity of the wire:

$$
\begin{aligned}
v_{\mathrm{c}}= & \frac{\varepsilon \varepsilon_{0}}{2 \eta} \cdot \frac{\ln (L / 2 r)+0.193}{\ln (2 L / r)-1} L E^{2} \\
& \pm \frac{g\left(\rho_{\mathrm{Ba}}-\rho_{\mathrm{He}}\right)}{2 \eta} \cdot(\ln (L / 2 r)+0.193) r^{2}
\end{aligned}
$$

The results of the calculations are shown in Fig. 11(c) and (d). We plot the charge $Q_{\mathrm{c}}$ in the units of elementary charge $e$ and the velocity versus the nanowire length $L$ for typical values of the electric field strength. The thickness of the nanowire is fixed at the value $2 r=10 \mathrm{~nm}$. The calculated charges and velocities are almost independent of the wire diameter.

The experimentally observed velocity distributions (Fig. 2) correspond to the nanowires with a length in the range of 0.1-2.0 $\mu \mathrm{m}$. The effect of gravity and buoyancy forces is negligible for such wires. The computed lifting field strength is even smaller than for spherical particles and decreases with increasing wire length. It does not exceed $30 \mathrm{~V} \mathrm{~cm}^{-1}$, i.e., every nanowire that is not attached to the electrode surface is expected to move upwards under the conditions of our experiments.

The charge acquired by a single nanowire is comparable to that of a spherical nanoparticle. The limit of $Q_{\mathrm{c}}=e$ is reached for the wires with $L \leq 480 \mathrm{~nm}$ at $E=1 \mathrm{kV} \mathrm{cm}^{-1}$ and $L \leq 800 \mathrm{~nm}$ at $E=400 \mathrm{~V} \mathrm{~cm}^{-1}$. The minimum observable velocity for the up-going wire at $E=1 \mathrm{kV} \mathrm{cm}{ }^{-1}$ is thus $v_{\text {min }}=19 \mathrm{~cm} \mathrm{~s}^{-1}$ and at $E=400 \mathrm{~V} \mathrm{~cm}^{-1} v_{\text {min }}=5 \mathrm{~cm} \mathrm{~s}^{-1}$. Both values are significantly larger than the smallest velocity observed at the corresponding electric field strength. The minimum of the particle velocity obtained in the experiment falls well within the range of low Reynolds number for the whole range of the electric field strength. It is therefore unlikely that the discrepancy arises because of the usage of eqn (11). We therefore suggest that some of the longer nanowires in contact with the electrode do not acquire the maximum charge as given by eqn (8) and therefore move slower than expected.

Another possible source of discrepancy is the effect of a vertical counterflow that may exist in superfluid $\mathrm{He}$ that is heated from above. The heat can be generated within the surface layer of liquid He due to the coalescence of metallic particles ${ }^{46}$ trapped at the surface, or due to the deceleration of fast atoms and larger particles coming from the ablation spot. As discussed in Section III C, the flow of the normal-fluid component from the heated area creates extra drag on the particle surface that is not balanced by the frictionless flow of the superfluid component. The counterflow will thus decelerate the particles moving upwards and accelerate those moving down.

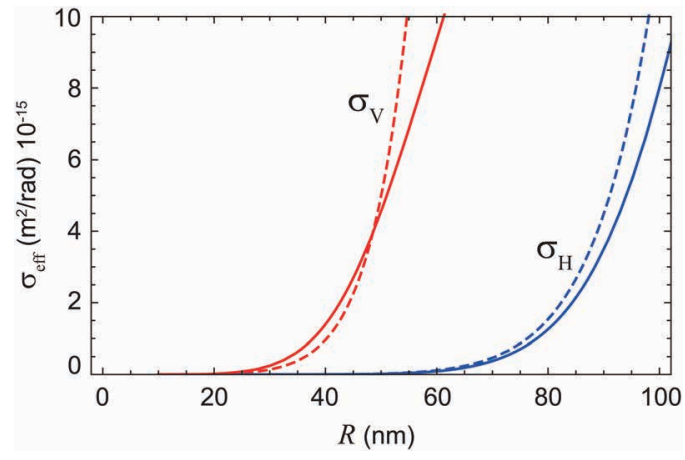

Fig. 12 Calculated differential cross sections for light scattering by $\mathrm{Ba}$ (solid lines) and $\mathrm{Cu}$ (dashed lines) nanoparticles at $\theta=\pi / 2, \lambda=480 \mathrm{~nm}$, vertical $\left(\sigma_{\mathrm{V}}\right.$, red curves) and horizontal $\left(\sigma_{\mathrm{H}}\right.$, blue curves) linear polarization.

\section{Discussion}

\section{A. Light scattering by nanoparticles}

We calculate the effective cross section for the light scattering by $\mathrm{Ba}$ and $\mathrm{Cu}$ nano-particles using the well-known formulae of Mie theory. ${ }^{43}$ The calculation uses the dielectric functions of Ba and $\mathrm{Cu}$ measured in ref. 44 and 45 , respectively.

The wavelength of the incident and scattered light is equal to that of our cw laser, $\lambda_{\text {las }}=480 \mathrm{~nm}$. The particles are assumed to have a spherical shape and the scattering angle is set to $\theta=\pi / 2$. The resulting differential cross sections for both materials, for vertical and horizontal light polarizations, $\sigma_{\mathrm{V}}$ and $\sigma_{\mathrm{H}}$ are plotted in Fig. 12 versus the particle radius, $R$.

The number of photons scattered by a single particle and reaching the camera sensor is calculated as

$$
n_{\mathrm{ph}}=\frac{W_{\text {las }} \lambda_{\text {las }}}{\pi r_{\text {las }}^{2} h c} \times \sigma_{\text {eff }} \delta \Omega \delta t
$$

Here, $W_{\text {las }}$ and $r_{\text {las }}$ are the laser power and the beam radius, $h$ is Planck's constant, $c$ is the speed of light, $\delta \Omega$ is a solid angle at which scattered light is collected and $\delta t$ is the exposure time.

For a vertically polarized laser beam with $W_{\text {las }}=100 \mathrm{~mW}$ and $r_{\text {las }}=3 \mathrm{~mm}$, a nano-particle as small as $R=20 \mathrm{~nm}$ scatters about 1000 photons per frame into the camera that should be sufficient for its detection. It is thus possible that the particles observed in liquid He via light scattering (Section III A) are the same as the spherical nanoparticles found in the SEM study (Section III D, Fig. 10). However, some sufficiently long segments of thinner nanowires, or their tangles may also scatter enough light and could be observed as point-like (diffractionlimited) objects.

\section{B. Charging at the electrode}

At a low applied voltage $E \approx 400 \mathrm{~V} \mathrm{~cm}^{-1}$, for both polarities the number of up-going particles is significantly smaller than that of the down-going particles. The corresponding distribution of the particle appearance times (see Fig. 3(a)) is strongly peaked $\approx 50 \mathrm{~ms}$ after the ablation laser pulse. The particles are created by the laser ablation above the surface, move down, then stick to the bottom electrode and do not return to the liquid surface. 
However, our calculations show that the lifting field strength for all relevant particles sizes and shapes is well below $400 \mathrm{~V} \mathrm{~cm}^{-1}$, therefore all particles should move up. As discussed in ref. 29 and 39, the contact between the electrode and the particle produces redistribution of charge on the electrode that reduces the electrostatic force acting on the particle. This effect leads to a certain increase in the lifting field strength that amounts to $17 \%$ for a spherical particle. For a wire, the effect becomes negligible with increasing aspect ratio $L / 2 r$. It is possible that for the small particles studied here some additional sticking mechanism becomes important, that is not captured by a purely macroscopic electrostatic model of ref. 29 and 39. As proposed in ref. 20 and 46, the energy released at a contact of two nanoparticles due to their coalescence may lead to a strong local heating and melting of the resulting particle encapsulated in a He micro-bubble. A similar effect may occur at the contact between the nanoparticle or a nanowire and the surface of the electrode. The local melting may lead to a "welding" of the nanowire end to the electrode surface.

At higher electric field strength, the numbers of sinking and rising particles become comparable. The sticking effect thus becomes insignificant at an electric field strength of $800 \mathrm{~V} \mathrm{~cm}^{-1}$.

The recharging of the particles at the lower electrode probably proceeds via the micro-breakdown in the gap between the electrode surface and approaching nanoparticle. ${ }^{29,37}$ The breakdown is expected there because of a very large local electric field enhancement in the vicinity of the nanoparticle. The breakdown is started at some critical particle-electrode distance that increases with the applied electric field strength. It is thus possible that at lower electric field strength the particle actually touches the electrode surface and has a higher probability to stick there. At larger electric field, the breakdown distance is large enough that the whole process of recharging is finished and the particle starts moving upwards before the contact with the electrode.

As discussed in Section IV C, many nanowires receive a charge significantly smaller than that predicted by the electrostatic model. This observation also supports the contact-less charging mechanism. The discharge between the particle and the electrode may terminate earlier than the maximum charge is transferred, when the distance between the particle and the electrode surface starts to increase. However, the reproducibility of the recharging process for the same particle seems to be very high. As is evidenced by the observations of the motion of a selected particle (see Fig. 4), many recharging events result in a very small variation of the particle velocity, typically less than $10 \%$.

\section{Charging at the free surface}

Charge carriers of both polarities - free electrons, $\mathrm{Ba}^{+}\left(\mathrm{Cu}^{+}\right)$and $\mathrm{He}^{+}$ions, charged $\mathrm{Ba}(\mathrm{Cu})$ clusters and nanoparticles - are created by the laser ablation in the He gas. Depending on the polarity of the applied electric field, carriers of one sign are driven towards the liquid He surface. Their motion in the gas phase is very much faster than in the liquid. So the charges are expected to arrive at the liquid surface within some microseconds after the ablation pulse. Typically, we shot ablation laser pulses at a $1 \mathrm{~Hz}$ repetition rate and the repetitive motion of particles continued during the whole time interval between the pulses. The effect is much more pronounced with $U_{\text {tar }}<0$, $U_{\text {plate }}>0$, when the negative charge carriers (free electrons) are driven towards the surface. This implies the presence of the negative charge at the surface, even at $1 \mathrm{~s}$ delay time with respect to the ablation laser pulse. It is well known that the free electrons cannot easily penetrate into liquid He because of the energy barrier of $\approx 1 \mathrm{eV}$ at the liquid surface. It is therefore not surprising that the negative charge may accumulate above the surface. On the other hand, all positive charge carriers, including $\mathrm{Ba}^{+}\left(\mathrm{Cu}^{+}\right)$and $\mathrm{He}^{+}$ions are expected to enter the liquid under the influence of the electrostatic force and therefore can not stay at the surface for a long time. Indeed, in this case the shuttle-like motion terminates typically after a single cycle and starts again after the next ablation pulse.

Independent of the applied electric field strength and polarity, the velocity of down-going particles, $v_{\text {down }}$, is lower than that of up-going particles, $v_{\text {up }}$, under the same conditions. We therefore conclude that the charge acquired by a particle at the free surface of liquid $\mathrm{He}$ is smaller than the charge obtained by the same particle in contact with the plate. The difference can be either due to the insufficient supply of charge at the free surface, or due to a slow charging process. In the latter case, the particle leaves the surface before the charging is completed.

\section{Filament growth}

The filaments are created as a result of the coalescence of nanoparticles and/or single ions and atoms produced by the laser ablation and injected into liquid helium. Most likely, the moving nano- and micro-particles observed in our video recordings represent the building blocks of those filaments and networks. The observed particle motion probably plays an important role in the filament growth process. Comparing the experimentally observed velocity distributions with the results of the calculations, we suggest that the observed moving particles are nanowire segments, several micrometers long, or nanowire bundles of the same length. It is not clear a priori where the network formation occurs predominantly: in the bulk, at the bottom electrode, or at the free surface.

Consider the experimental arrangement with the negative potential of the plate and positive potential of the ablation target. Under these conditions, the shuttle-like motion of charged particles terminates after a single cycle due to the absence of positive charge carriers at the free surface of the liquid. A negatively charged particle trapped under the free surface collects some number of positive ions coming from the ablation plume, travels to the bottom electrode, recharges, and returns to the surface. It stays at the surface till the next ablation pulse. It therefore spends most of the time at the surface. On the other hand, at the opposite polarity of the electric field, free electrons easily accumulate at the free surface and the shuttle-like motion of metallic particles goes on all the time. The particles spend most of the time moving through the 
liquid bulk, or sticking at the bottom electrode. In this case, any surface-related mechanism of the network formation will be suppressed. In the experiment, the filaments and networks are observed only with the negative potential of the grid and positive potential of the ablation target. We therefore suggest that the network formation occurs mostly at the surface and that the active shuttle-like motion of particles leads to its suppression.

On every ablation pulse, filaments of various sizes temporarily leave the surface and make one run towards the bottom electrode and back. Occasionally, some of them stick to the surface of the electrode and remain there. Their growth may continue due to the attachment of other shuttle-particles. Some of them eventually evolve into very large tree-like structures strongly pinned at the electrode surface (see Fig. 5(b)). Only an electric field in excess of $1 \mathrm{kV} \mathrm{cm}^{-1}$ is able to detach them from the electrode. Following the authors of ref. 20 and 46 we suggest that the nanowires and nanoparticles may become molten at the point of their contact. After the resolidification, the joint between two nanowires or between the nanowire and the electrode becomes really strong.

Our video recordings show numerous events, when two particles or large filaments touch each other and stick together. This is possible if the two particles have charges of different polarities, or one or both of them are electrically neutral. We observe such events mostly at the free surface, with the negatively charged filaments or fragments of networks disconnected from the electrode. Before sticking together, the filaments lose their charge due to the flux of positive charge carriers coming from above. Most likely, the sticking becomes possible when one part of the network is still charged and another has already neutralized. Such events certainly contribute to the growth of the large networks and "trees".

\section{Summary}

In summary, we have extended earlier studies of nanowire and network formation in superfluid He to the new system involving a free surface of superfluid $\mathrm{He}$ in a vertical static electric field. We observe a preferential formation of the nanowires and mesoscopic networks at the surface that is strongly influenced by the polarity and the strength of the applied electric field. The observations suggest that the free surface of liquid $\mathrm{He}$ in a vertical electric field acts as a very efficient two-dimensional trap for various electrically charged impurity particles and thus strongly enhances their coalescence and network formation. Our results closely reproduce those obtained earlier in bulk superfluid He without the electric field. ${ }^{1,6,8,10,12}$ There is also a close analogy between our observations and the results of the experiments involving superfluid He microdroplets. ${ }^{14-16}$

It was argued that in bulk superfluid He and in He microdroplets the nanowires are formed due to the trapping of the impurity atoms at the cores of quantized vortices. Most likely, quantized vortices are also present in our sample cell under the typical experimental conditions. They may be responsible for the formation of vertically oriented filaments attached to the bottom electrode and other parts of the sample cell. However, vortices are always oriented orthogonally to the free surface of the liquid, at least in the vicinity of that surface. ${ }^{18}$ The surfacebound nanowires and networks observed in our experiment are thus oriented orthogonally to the vortices and their growth most likely is not induced by that mechanism. We believe that the two-dimensional trapping of particles by the free surface of liquid $\mathrm{He}$ is at the origin of the surface nanowire and network formation. We propose that a more detailed comparison of the structures and shapes of the networks and "ropes" produced at the surface and in the bulk may lead to a better understanding of the role of the $1 \mathrm{D}$ and $2 \mathrm{D}$ trapping in the nano-network formation.

\section{Acknowledgements}

We acknowledge the help from D. Inoue who operated SEM machine. This work was supported by JSPS KAKENHI grant No. 24000007.

\section{References}

1 A. Fujisaki, K. Sano, T. Kinoshita, Y. Takahashi and T. Yabuzaki, Phys. Rev. Lett., 1993, 71, 1039.

2 M. Arndt, PhD thesis, Ludwig Maximilians Universität München, Max-Planck-Institut für Quantenoptik, Garching, MPQ-Report 197, 1995.

3 G. P. Bewley, D. P. Lathrop and K. R. Sreenivasan, Nature, 2006, 441, 588.

4 E. B. Gordon, R. Nishida, R. Nomura and Y. Okuda, JETP Lett., 2007, 85, 581.

5 E. B. Gordon and Y. Okuda, Low Temp. Phys., 2009, 35, 209.

6 P. Moroshkin, V. Lebedev, B. Grobety, C. Neururer, E. B. Gordon and A. Weis, Europhys. Lett., 2010, 90, 34002.

7 E. B. Gordon, A. V. Karabulin, V. I. Matyushenko, V. D. Sizov and I. I. Khodos, Low Temp. Phys., 2010, 36, 590.

8 V. Lebedev, P. Moroshkin, B. Grobety, E. Gordon and A. Weis, J. Low Temp. Phys., 2011, 165, 166.

9 E. B. Gordon, A. V. Karabulin, V. I. Matyushenko, V. D. Sizov and I. I. Khodos, Appl. Phys. Lett., 2012, 101, 052605.

10 E. B. Gordon, A. V. Karabulin, V. I. Matyushenko, V. D. Sizov and I. I. Khodos, J. Low Temp. Phys., 2013, 172, 94.

11 E. B. Gordon, A. V. Karabulin, A. A. Morozov, V. I. Matyushenko, V. D. Sizov and I. I. Khodos, J. Phys. Chem. Lett., 2014, 5, 1072.

12 E. Gordon, A. Karabulin, V. Matyushenko, V. Sizov and I. Khodos, Phys. Chem. Chem. Phys., 2014, 16, 25229.

13 E. B. Gordon, A. V. Karabulin, V. I. Matyushenko and I. I. Khodos, J. Phys. Chem. A, 2015, 119, 2490.

14 L. F. Gomez, E. Loginov and A. F. Vilesov, Phys. Rev. Lett., 2012, 108, 155302.

15 P. Thaler, A. Volk, F. Lackner, J. Steurer, D. Knez, W. Grogger, F. Hofer and W. E. Ernst, Phys. Rev. B: Condens. Matter Mater. Phys., 2014, 90, 155442. 
16 E. Latimer, D. Spence, C. Feng, A. Boatwright, A. M. Ellis and S. Yang, Nano Lett., 2014, 14, 2902.

17 A. Volk, P. Thaler, D. Knez, A. W. Hauser, J. Steurer, W. Grogger, F. Hofer and W. E. Ernst, Phys. Chem. Chem. Phys., 2016, 18, 1451.

18 R. J. Donnelly, Quantized vortices in helium II, Cambridge University Press, Cambridge, 1991.

19 E. J. Yarmchuk, M. J. V. Gordon and R. E. Packard, Phys. Rev. Lett., 1979, 43, 214.

20 E. B. Gordon, A. V. Karabulin, V. I. Matyushenko, V. D. Sizov and I. I. Khodos, Chem. Phys. Lett., 2012, 519-520, 64.

21 N. G. Berloff and P. H. Roberts, Phys. Rev. B: Condens. Matter Mater. Phys., 2000, 63, 024510.

22 D. Kivotides, C. F. Barenghi and Y. A. Sergeev, Phys. Rev. B: Condens. Matter Mater. Phys., 2008, 77, 014527.

23 C. R. Knutson, K. V. Edmond, M. T. Tuominen and A. D. Dinsmore, J. Appl. Phys., 2007, 101, 013706.

24 M. Hase, S. N. Watanabe and K. Yoshikawa, Phys. Rev. E: Stat., Nonlinear, Soft Matter Phys., 2006, 74, 046301.

25 T. Kurimura, M. Ichikawa, M. Takinoue and K. Yoshikawa, Phys. Rev. E: Stat., Nonlinear, Soft Matter Phys., 2013, 88, 042918.

26 C. P. Lee, H. C. Chang and Z. H. Wei, Appl. Phys. Lett., 2012, 101, 014103.

27 A. M. Drews, C. A. Cartier and K. J. M. Bishop, Langmuir, 2015, 31, 3808.

28 A. M. Drews, M. Kowalik and K. J. M. Bishop, J. Appl. Phys., 2014, 116, 074903.

29 R. Tobazeon, J. Phys. D: Appl. Phys., 1996, 29, 2595.

30 L. Dascalescu, R. Tobazeon and P. Atten, J. Phys. D: Appl. Phys., 1995, 28, 1611.
31 L. Dascalescu, A. Samuila and R. Tobazeon, J. Electrost., 1996, 37, 173.

32 L. Dascalescu, M. Mihailescu and R. Tobazeon, IEEE Trans. Ind. Appl., 1998, 34, 66.

33 C. Choi, K. Yatsuzuka and K. Asano, IEEE Trans. Ind. Appl., 2001, 37, 785.

34 M. Hara, H. Nakagawa, T. Shinohara and J. Suehiro, IEEE Trans. Dielectr. Electr. Insul., 2002, 9, 910.

35 M. Hara, Y. Maeda, N. Nakagawa, J. Suehiro and S. Yamada, IEEE Trans. Dielectr. Electr. Insul., 2006, 13, 470.

36 L. Musinski, T. Liu, B. Gilchrist and A. Gallimore, J. Electrost., 2009, 67, 54.

37 K. Gloos, W. Schoepe, J. T. Simola and J. T. Tuoriniemi, Cryogenics, 1992, 32, 791.

38 M. Shindo, A. Samarian and O. Ishihara, JPS Conf. Proc., 2014, 1, 015049.

39 N. J. Felici, Rev. Gen. Electr., 1966, 75, 1145.

$40 \mathrm{~J}$. Wilks, The properties of liquid and solid helium, Clarendon Press, Oxford, 1967.

41 B. Eck, Einführung in die technische Strömungslehre, Springer, Berlin, 1935.

42 H. Anis and K. D. Srivastava, IEEE Trans. Electr. Insul., 1981, 16, 327.

43 H. C. van de Hulst, Light scattering by small particles, Dover Publications, New York, 1981.

44 J. G. Endriz and W. E. Spicer, Phys. Rev. B: Solid State, 1970, 2, 1466.

45 P. B. Johnson and R. W. Christy, Phys. Rev. B: Solid State, 1972, 6, 4370.

46 E. B. Gordon, A. V. Karabulin, V. I. Matyushenko, V. D. Sizov and I. I. Khodos, J. Exp. Theor. Phys., 2011, 112, 1061. 